\title{
DO COMPUTERISED VISUAL FIELDS AND AUTOMATED OPTIC DISC ANALYSIS ASSIST IN THE CHOICE OF THERAPY IN GLAUCOMA?
}

\author{
FREDERICK S. MIKELBERG \\ Vancouver, Canada
}

\begin{abstract}
SUMMARY
The choice of therapy in patients with glaucoma is determined by the presence of damage in the visual field or optic disc or by change in the appearance of the optic disc or visual field over time. Computerised visual fields and automated optic disc analysis can assist in this determination. Automated optic disc analysis permits the measurement of neuroretinal rim area which is more closely related to the presence of pathology than cup/disc ratio. Simple ophthalmoscopic techniques may also be used to estimate the size of the neuroretinal rim area. Automated perimetry allows accurate diagnostic information to be obtained in a reliable manner. This permits easier diagnosis of glaucoma as well as easier long term follow up for change. The availability of automated techniques in ophthalmology has helped the clinician in the choice of therapy. The use of astute clinical judgement remains however of the utmost importance.
\end{abstract}

Physicians are faced with two major issues in the diagnosis and treatment of patients with glaucoma. In the "glaucoma suspect" with normal visual fields, normal optic nerves and elevated intraocular pressure, the decision to initiate therapy is usually based on the number of risk factors. The presence of damage either in the visual field or in the optic disc necessitates treatment. In individuals with glaucomatous damage the choice of therapy includes medical, laser or surgical therapy, each with its own benefits and risks.

Computerised visual fields and automated optic disc analysis can assist in the choice of therapy in individuals with elevated intraocular pressure and no apparent damage. Detailed examination of the visual field and optic disc may reveal the presence of pathology which would otherwise be missed. Individuals with glaucomatous damage may have more accurate determination of progression with the use of computerised and automated systems. Our

From Department of Ophthalmology. University of British Columbia.

Correspondence to: Dr. F. S. Mikelberg, 2550 Willow Street, Vancouver, BC Canada V5Z 3N9. choice of therapy is therefore largely determined by the presence of risk factors, the presence of acquired pathology and documented progression in either visual field or optic disc parameters.

Because the optic nerve is the site of damage in this disease, accurate techniques of examination will improve diagnosis of individuals with glaucoma as well permit the accurate determination of progression. Intraocular pressure fluctuates but the status of the optic nerve can usually be determined with certainty. The techniques used to examine the optic nerve range from simple to complex and vary in cost from relatively inexpensive to quite expensive. The simplest method of examination is the use of the direct ophthalmoscope. It allows rapid, easy examination of the optic nerve but because it is a monocular instrument the disc cannot be evaluated for topographical change. All inferences of topography are based on monocular depth clues. The use of a contact lens in combination with a slit lamp permits binocular examination of the optic disc. This therefore allows stereo evaluation of the disc and the topographical contours can be determined. Photographs can be obtained. Standard photographs are useful and more useful yet are stereo photographs which again allow a stereoscopic record of the optic disc to be obtained. These records are invaluable, both as baseline data to evaluate change and to evaluate subtle details of the optic disc which may have been missed in the clinical examination. Stereo photographs can be analysed in a semi-automated manner using planimetry. ${ }^{1}$ With this technique a record of the neuroretinal rim area can be obtained. By adding axial length measurement obtained with ultrasound and the patient's refractive error, these area measurements can be corrected so that the magnification induced by the optical system of the eye is corrected. ${ }^{2}$ Measurements can be obtained with the use of automated optic disc analysers which have not yet proven to be clinically useful. ${ }^{3,4}$

The evaluation of the cup to disc ratio has historically been the most common method of determining the health of the optic nerve. Unfortunately it suffers from a number of drawbacks. There is a lack of inter and intra observer

Eye (1992) 6, 47-49 
repeatability. ${ }^{5}$ There is also the problem that the cup to disc ratio is dependent on the optic disc size. ${ }^{6}$ Normal individuals with large discs will have larger cup to disc ratios and those with smaller discs will have smaller cup to disc ratios. Nevertheless, there are a number of findings which provide clues to the health of the optic disc. Cup to disc ratio asymmetry between eyes is quite suspicious. Presence of an optic disc haemorrhage is always suspicious as these haemorrhages rarely occur in normal individuals. The presence of notching and vertical elongation of the cup is also of importance.

Because the cup to disc ratio is so dependent on the optic disc size, we have focused more interest on the evaluation of the neuroretinal rim area. This is the region containing the axons existing the eye. Studies have revealed that the rim area correlates well with a number of findings. The rim area is the best optic disc predictor of diagnostic category between normals, glaucoma suspects and patients with glaucoma. There is unfortunately however a large amount of overlap amongst the different groups. The rim area correlates very well with other anatomical measures such as photographic evaluation of the retinal nerve fibre layer. ${ }^{7}$ The rim area also correlates well with the visual field status. ${ }^{8}$ It particularly correlates well with the overall sensitivity of the field as measured in mean deviation.

Unfortunately the rim area is also related to the disc area. Studies have shown that eyes with larger optic discs have larger rim areas and vice versa. ${ }^{9,10,11}$ Because of this, the evaluation of the rim area cannot be made in isolation but must be compared to nomograms which take into account the size of the optic disc. This relationship becomes particularly important in those individuals with very small optic discs. In these discs, there is no visible cup present in the normal situation. In these eyes, the presence of a cup may herald significant disc damage. ${ }^{12}$ Disc damage may even be occurring while the disc still appears normal. These types of eyes will often show visual field loss prior to discernable disc damage being present. At the other end of the spectrum are eyes with large optic discs. These eyes will have quite large cups and may still be normal. It is important to note however that eyes with large discs may get glaucoma and therefore large cups may become even larger indicating the presence of damage.

We can assess the size of the optic disc with the direct ophthalmoscope. ${ }^{13}$ The projection of the small aperture of the Welsh Allan direct ophthalmoscope is about 10 to $20 \%$ less than the size of a normal optic disc. When examining a patient in which the optic disc is much smaller than the projection of the ophthalmoscope, one must be suspicious that significant damage could occur in the absence of marked cupping. Conversely, when the projection of the ophthalmoscope is significantly smaller than the optic disc, these eyes can have significant cupping and remain normal.

The accurate assessment of the optic disc is essential in diagnosing glaucoma as well as assessing the adequacy of therapy. It is therefore of the utmost importance in determining the choice of therapy both in determining the time of initiation of therapy as well the adequacy of therapy and whether therapeutic modalities need to be changed. Automated techniques are useful because they may permit accurate determination of the presence of pathology when measuring neuroretinal rim area as well as possibly permitting accurate determination for change. They are however not essential as semi-automated techniques such as manual planimetry can currently permit determination of the same information. Most importantly, with the simple use of the direct ophthalmoscope we can detect the presence of notching and haemorrhage, we can determine whether significant asymmetry is present, we can estimate the size of the optic disc and therefore determine the significance of the size of the cupping. All of the above are useful in the choice of therapy.

The second modality involved in the determination of the presence of glaucoma as well as the adequacy of treatment is detailed visual field analysis. Although manual techniques can be performed quite accurately, they suffer from a number of drawbacks including the problem of inter-technician variability as well as variability within the same technician on different days. Automated visual field machines have permitted accurate perimetry to be performed in any office at a relatively low cost. Although non-automated perimetry allows accurate diagnostic information to be obtained only the development of automated fields has permitted detailed statistical analysis of threshold data. For diagnosis, automated visual fields are useful in the determination of normalcy. Visual field indices provide a convenient summary of the overall characteristics of the visual field including determination of localised versus non-localised loss as well as the presence of short term fluctuation. ${ }^{14}$ The development of probability maps for the visual field allow the accurate determination of the estimated probability of a point with a given threshold occuring in a normal individual. ${ }^{15}$ Statistical comparison of the superior and inferior hemifield portions also provides sensitive indications of abnormality. ${ }^{16}$ These are only possible with the use of automated perimetry.

The determination of change in a visual field has always been difficult. This is inherently difficult because of the presence of long term fluctuation which is even greater in individuals with glaucoma. ${ }^{17}$ Automated visual fields have now permitted the use of this data in sophisticated programs to compare each location on a point by point basis with a number of baseline visual fields to determine whether the amount of change in an individuals point exceeds that which can be expected to occur due to long term fluctuation. ${ }^{18}$ This also has been determined through the careful analysis of visual fields belonging to patients with glaucoma.

The availability of automated visual fields therefore permits the clinician to make more accurate diagnoses as to the presence of pathology as well as to the development of change in a given patient's visual field. This allows an 
easier determination of the need for therapy and also an easier determination for the modification of therapy in individuals who may be progressing.

\section{REFERENCES}

1. Airaksinen PJ, Drance SM, Schulzer M: Neuroretinal rim area in early glaucoma. Am J Ophthalmol 1985, 99: 1-4.

2. Littman $\mathrm{H}$ : Zur verstimmung der Wahren grosse eines Objecktes auf dem hintergrund des leben auges. Klin Monatsbl Augenheilkd 1982, 180: 286-9.

3. Mikelberg FS, Airaksinen PJ, Douglas GR, Schulzer M, Wijsman K: The correlation between optic disc topography measured by the video-ophthalmograph (Rodenstock Analyzer) and clinical measurement. Am J Ophthalmol 1985, 100: 417-19.

4. Caprioli J, Klingbeil U, Sears M, Pope B: Reproducibility of optic disc measurements with computerized analysis of stereoscopic video images. Arch Ophthalmol 1986, 104: 1035-9.

5. Lichter PR: Variability of expert observers in evaluating the optic disc. Trans Am Ophthalmol Soc 1976, 75: 532-72.

6. Balazsi AG, Drance SM, Schulzer M, Douglas GR: Neuroretinal rim area in suspected glaucoma and early chronic open angle glaucoma. Arch Ophthalmol 1984, 102: 1011-14.

7. Airaksinen PJ and Drance SM: Neuroretinal rim area and retinal nerve fibre layer in glaucoma. Arch Ophthalmol 985, 103: 203-4.

8. Airaksinen PJ, Drance SM, Douglas GR, Schulzer M: Neuroretinal rim area and visual field indices in glaucoma. Am J Ophthalmol 1985, 99: 107-110.

9. Jonas JB, Gusek GC, Guggenmoos-Holzmann I, Naumann
$\mathrm{GOH}$ : Correlations of the neuroretinal rim area with ocular and general parameters in normal eyes. Ophthalmic Res 1988, 20: 298-303.

10. Britton RJ, Drance SM, Schulzer M, Douglas GR, Mawson DK: The area of the neuroretinal rim of the optic nerve in normal eyes. Am J Ophthalmol 1987, 103: 497-504.

11. Caprioli $\mathrm{J}$ and Miller JM: Optic disc rim area is related to disc size in normal subjects. Arch Ophthalmol 1987, 105: 1683-5.

12. Jonas JB, Gusek GC, Naumann GOH: Optic disc morphometry in chronic primary open angle glaucoma, I morphometric intrapapillary characteristics. Graefe's Arch Clin Exp Ophthalmol 1988b, 226: 522-30.

13. Gross P: Fundus measurements with the direct ophthalmoscope: I. Light spot size at the retina. (Submitted for publication).

14. Flammer J, Drance SM, Augustiny L, Funkhouser A: Quantification of Glaucomatous Visual Field Defects with Automated Perimetry. Invest Ophthalmol Vis Sci 1985, 26: 176-81.

15. Heijl A, Lindgren G, Olsson J, Asman P: Visual Field Interpretation with Empiric Probability Maps. Arch Ophthalmol 1989, 107: 204-8.

16. Sommer A, Enger C, Witt K: Screening for Glaucomatous Visual Field Loss with Automated Threshold Perimetry. Am J Ophthalmol 1987, 103: 681-4.

17. Drance SM, Flammer J, Zulauf M: Differential Light Threshold: The Short and Long Term Fluctuation in Patients with Glaucoma, Normal controls and Patients with Suspected Glaucoma. Arch Ophthalmol 1984, 102: 704-6.

18. Heijl A, Lindgren A, Lindgren G: Test-retest Variability in Glaucomatous Visual fields. Am J Ophthalmol 1989, 108: $130-5$. 